Canadian University Music Review

Canadian University Music Review

Revue de musique des universités canadiennes

\title{
Une démarche esthésique, ou le temps retrouvé : présentation critique de l'ouvrage Les écritures du temps de Michel Imberty
}

\section{Leiling Chang Melis}

Volume 20, numéro 2, 2000

URI : https://id.erudit.org/iderudit/1014459ar

DOI : https://doi.org/10.7202/1014459ar

Aller au sommaire du numéro

\section{Éditeur(s)}

Canadian University Music Society / Société de musique des universités canadiennes

ISSN

0710-0353 (imprimé)

2291-2436 (numérique)

Découvrir la revue

Citer cet article

Chang Melis, L. (2000). Une démarche esthésique, ou le temps retrouvé : présentation critique de l'ouvrage Les écritures du temps de Michel Imberty. Canadian University Music Review / Revue de musique des universités canadiennes, 20(2), 80-93. https://doi.org/10.7202/1014459ar
Résumé de l'article

L'ouvrage Les écritures du temps (1981) de Michel Imberty est d'une importance capitale pour la musicologie contemporaine. De la psychologie expérimentale à la psychanalyse, Imberty développe une approche perceptive du style musical dont le but est l'interprétation de la musique en tant que symbolique du temps. Sa démarche est explicable à partir de la sémiologie musicale, qui en clarifie les principes. Imberty construit, avec précision et intelligence, une méthodologie pour l'observation du temps musical, et cela constitue son grand apport à la musicologie contemporaine. Cet essai cherche à mettre en relief les notions et postulats fondamentaux de sa pensée.
All Rights Reserved ( C Canadian University Music Society / Société de musique des universités canadiennes, 2000
Ce document est protégé par la loi sur le droit d'auteur. L'utilisation des services d'Érudit (y compris la reproduction) est assujettie à sa politique d'utilisation que vous pouvez consulter en ligne.

https://apropos.erudit.org/fr/usagers/politique-dutilisation/ 


\title{
UNE DÉMARCHE ESTHÉSIQUE, OU LE TEMPS RETROUVÉ : PRÉSENTATION CRITIQUE DE L'OUVRAGE LES ÉCRITURES DU TEMPS DE MICHEL IMBERTY
}

\author{
Leiling Chang Melis
}

\section{INTRODUCTION}

Auteur de plusieurs articles et ouvrages, Michel Imberty occupe une place capitale dans la musicologie contemporaine ${ }^{1}$. Consacrant sa démarche à l'étude de la sémantique musicale, et plus spécifiquement aux problèmes du temps musical, comme les structures temporelles des œuvres musicales et la représentation psychologique du temps, Imberty fait converger des lignées diverses du savoir. En réunissant deux branches distinctes de la psychologie, soit la psychologie expérimentale et la psychanalyse, il fait aussi le parcours de plusieurs approches théoriques, essentiellement la phénoménologie, l'herméneutique et la sémiologie. Les écritures du temps, deuxième et dernier volume d'une étude poussée sur la sémantique psychologique de la musique, prolonge une approche esthésique du style musical déjà annoncée et élaborée dans Entendre la musique, le volume précédent, qui est entièrement consacré à la sémantique musicale et à la psychologie expérimentale, tout en faisant basculer le discours vers une herméneutique d'ordre psychanalytique, érigée sur l'hypothèse suivante :la musique est une symbolique du temps;l'œuvre musicale, forme du temps, se détache de l'écoulement temporel réel dont la fin n'est que le vieillissement et la mort, et vit dans une espèce de présent psychologique éternel où les vécus du Moi sont reflétés et synthétisés. L'art musical, possibilité

\footnotetext{
${ }^{1}$ Michel Imberty est professeur à l'Université de Paris X-Nanterre et a été président de cet établissement de 1994 à 1997. Il a une formation à la fois musicologique, philosophique et psychologique. Il a édité avec Françoise Escal La musique au regard des sciences humaines et des sciences sociales : actes du colloque, Maison des sciences de l'homme, Paris, 10 et 11 février 1994, Logiques sociales, série " Musiques et champ social » (Paris : L'Harmattan, 1997). Il est surtout connu pour ses livres Entendre la musique : sémantique psychologique de la musique, Collection « Psychismes » (Paris:Dunod,1979),xvi,236 p. (d'abord paru comme thèse de doctorat de l'auteur, Université de Paris X-Nanterre); Les écritures du temps : sémantique psychologique de la musique, tome 2, Collection « Psychismes" (Paris : Dunod, 1981), xvi, 274 p. Voir aussi ses articles « La Cathédrale engloutie de Claude Debussy : de la perception au sens », Revue de musique des universités canadiennes, $\mathrm{n}^{\circ} 6$ (1985) : 90-160; « De la perception du temps musical à sa signification psychologique : à propos de "la Cathédrale engloutie" de Claude Debussy ", Analyse musicale, n" 6 (janvier 1987) : 28-37; « Le style musical : aspects esthésiques et aspects poḯtiques ", Analyse musicale, $\mathrm{n}^{\circ} 32$ (juillet 1993) : 14-18.
} 
psychologique de contourner l'écoulement du temps et d'échapper momentanément au vieillissement et à la mort, réaliserait alors ce que Marcel Proust a si génialement remarqué à propos des processus psychiques qui, par l'association des vécus différents, permettent de «sentir » le passé, de le "vivre », comme s'il s'agissait du présent, d'effacer toute frontière temporelle et enfin de situer l'être humain dans une dimension du temps, ou du hors-temps, qui évacue totalement la préoccupation de la mort :

[...] l'être qui alors goûtait en moi cette impression la goûtait en ce qu'elle avait de commun dans un jour ancien et maintenant, dans ce qu'elle avait d'extra-temporel, un être qui n'apparaissait que quand, par une de ces identités entre le présent et le passé, il pouvait se trouver dans le seul milieu où il peut vivre, jouir de l'essence des choses, c'est-à-dire en dehors du temps. Cela expliquait que mes inquiétudes au sujet de ma mort eussent cessé $[\ldots]^{2}$.

Le geste herméneutique d'Imberty est rigoureux : de l'exactitude et de la précision de l'enquête réalisée dépend la valeur de l'interprétation finale de la signification intrinsèque. Prenant des distances devant le relativisme contemporain excessif, il touche un des points faibles de l'herméneutique moderne :

[...] le décryptage que proposent en exemple la plupart des travaux herméneutiques sur la musique, repose constamment sur des références aux éléments extra-musicaux fournis par la biographie du compositeur, ou, dans le meilleur des cas, par le texte littéraire d'une musique à support vocal (p. 166) ${ }^{3}$.

Ce qui est aussi le cas des interprétations psychanalytiques : «L'erreur de la plupart des travaux psychanalytiques, en littérature comme en musique [...] consiste à partir de la biographie et non de l'œuvre » (p. 80). Pour Imberty, au contraire, il est absolument indispensable de montrer « le primat de la forme comme expression symbolique »(p.167). Toujours à la recherche de significations intrinsèques, il prolonge un questionnement qui était déjà présent dans le psychologisme du philosophe allemand Wilhelm Dilthey (1833-1911), soit la révélation de la vie psychique à travers les œuvres.

La conception de la musique en tant que phénomène de sens rapproche Imberty de la phénoménologie. Les notions de conscience intentionnelle et d'objet intentionnel sont à la base de sa démarche. Le lien avec le philosophe allemand Edmund Husserl (1859-1938) est clair et Imberty le révèle lui-même. Cependant, la correspondance avec les propos du philosophe polonais Roman - Ingarden (1893-1970), dont l'apport à la phénoménologie de la musique est capital, nous frappe, car la traduction française des textes d'Ingarden date seulement de 1989. Au delà des notions phénoménologiques fondamentales de

\footnotetext{
${ }^{2}$ Marcel Proust, À la recherche du temps perdu : Le temps retrouvé II (Paris : Gallimard, 1927), 13.

${ }^{3}$ Toutes les références à l'ouvrage Les écritures du temps de Michel Imberty sont données entre parenthèses dans le texte plutôt qu'en note.
} 
conscience et d'objet intentionnels, le lien avec Ingarden touche les piliers de la vision qu'Imberty élabore sur la musique : le temps, le mouvement, les concepts de macrostructure et de relation d'ordre, le style en tant que structure temporelle, entre autres. Et cela sans toucher le problème de l'existence de l'œuvre musicale, qui se résout de la façon suivante chez Ingarden : l'œuvre musicale devient objet, elle existe, parce qu'elle est un Tout unifié, une totalité, une structure temporelle qui se détache de la réalité; son existence est proportionnelle à son intégrité dans le temps.

Imberty n'est donc pas le premier chercheur à aborder la musique du point de vue de son existence dans le temps. Déjà en 1854, dans Vom MusikalischSchönen (Du beau dans la musique), Eduard Hanslick mettait en relief l'idée suivante : "Que contient donc la musique? Pas autre chose que des formes sonores en mouvement ${ }^{4} »$. Pendant la première moitié $\mathrm{du} \mathrm{XX}^{\mathrm{e}}$ siècle, plusieurs théoriciens de la musique ont mis l'accent sur l'importance du temps et du mouvement en tant que notions essentielles pour l'étude de la musique. Parmi eux, Ingarden et Theodor W. Adorno (1903-69), le philosophe et musicologue allemand, en ont développé des réflexions originales ouvrant un terrain d'exploration révélateur pour les recherches musicologiques. Pour Ingarden, par exemple : " Le premier moment non acoustique de l'œuvre musicale, intimement uni avec les entités sonores en développement et en succession, est la structure temporelle, immanente à l'œuvre en $\mathrm{soi}^{5}$ », tandis que «l'apparition du mouvement » constitue le deuxième moment non acoustique ${ }^{6}$. Cependant, Adorno tend plutôt vers une interprétation psychologique du temps musical. La musique est pour lui la « décoration du temps vide ${ }^{7}$ ». Il s'explique plus loin : «[...] les hommes craignent le temps et inventent pour cela des métaphysiques compensatoires du temps $[\ldots]^{8}$.» Or, Ingarden n'a pas seulement réfléchi sur la question du temps et du mouvement, mais il a signalé tout ce qu'il restait à faire pour clarifier l'essence de l'art musical :

Afin de décrire plus précisément les variantes de l'organisation temporelle possible dans l'œuvre musicale, on devrait effectuer une analyse exhaustive d'une série d'œuvres, ce que nous ne pouvons pas nous permettre ici. Une telle analyse, à ma connaissance, n'a encore jamais été réalisée. Elle appartient de droit à la musicologie, et non à l'explication phénoménologique générale de l'œuvre musicale, telle que nous la proposons ici. L'accomplissement de cette tâche scientifique prêterait d'abord à nos exposés des cas concrets en grand nombre, et elle fournirait à nos réflexions une confirmation empirique souhaitable 9 .

${ }^{4}$ Eduard Hanslick, Du beau dans la musique, trad. par Charles Bannelier, revue et complétée par Georges Pucher, Collection « Musique/Passé/Présent » (Paris : Christian Bourgois, 1986), 94.

${ }^{5}$ Roman Ingarden, Qu'est-ce qu'une æuvre musicale?, trad. par Dujka Smoje, Collection «Musique/Passé/Présent » (Paris : Christian Bourgois, 1989), 109.

${ }^{6}$ Ibid., 112.

${ }^{7}$ Theodor Adorno, Introduction à la sociologie de la musique : douze conférences théoriques (Paris : Contrechamps, 1994), 53.

8 Ibid., 54.

${ }^{9}$ Ingarden, Qu'est-ce qu'une æuvre musicale?, 110. 
C'est sans doute Imberty qui réalise cette tâche scientifique et synthétise la stratégie réflexive d'Ingarden et les interprétations psychologiques d'Adorno.

La position d'Imberty par rapport à la sémiologie musicale, et surtout à propos de la tripartition soutenue par le linguiste Jean Molino et le musicologue JeanJacques Nattiez ${ }^{10}$, est révélatrice. Le modèle tripartite consiste dans l'établissement de trois niveaux distincts du phénomène musical : (1) le niveau poḯtique, qui concerne les stratégies de création, (2) le niveau neutre, qui concerne les configurations immanentes des œuvres musicales, et (3) le niveau esthésique, qui concerne les processus perceptifs. Molino et Nattiez insistent sur la séparation des niveaux, sur la nécessité de la réalisation d'études indépendantes et autonomes de chacun des niveaux, avec des méthodes d'analyse précises et distinctes, dans le but de rendre compte de la complexité du fait musical total. La position critique d'Imberty devant le modèle tripartite explique l'ensemble de sa démarche; elle dévoile le postulat clé qui justifie chacune de ses réflexions. À l'encontre de ce que Molino et Nattiez affirment, Imberty défend l'unité des niveaux poḯtique, neutre et esthésique par l'existence de processus psychiques universels ${ }^{11}$ qui dirigent autant la création d'une œuvre que sa perception. La perception d'une œuvre musicale serait alors en correspondance avec sa structure stylistique et avec les intentions du créateur; découvrir les processus psychiques universels mis en jeu dans des relations de «résonance » entre l'auditeur et l'œuvre, et entre l'œuvre et le compositeur, serait l'objectif de l'interprétation psychanalytique. C'est pour cela que, plutôt qu'une réalité sémiologique, le style est pour Imberty une réalité psychologique.

\section{Isomorphisme des trois niveaux de la tripartition}

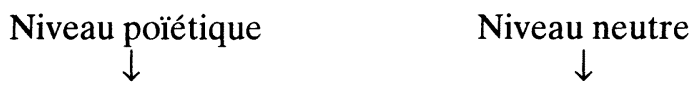

$\underset{\downarrow}{\text { Niveau esthésique }}$

Processus psychiques universels permettant des relations de résonance entre les trois niveaux. Universalité des processus de représentation symbolique.

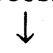

Possibilité de communication, transmission du message

La possibilité de déduire le fonctionnement de chacun des niveaux à partir de l'étude poussée de l'un d'eux se dégage de cette vision du processus musical. Imberty fait le choix du niveau esthésique, car le sens se réalise pleinement dans la perception, et à partir de l'examen du style en tant que réalité perceptive, sa démarche se déplace vers ce qui est perçu du niveau neutre, c'est-à-dire, vers ce qui, au niveau neutre, a conditionné l'écoute, pour finalement interpréter

\footnotetext{
${ }^{10}$ Jean Molino, «Fait musical et sémiologie de la musique », Musique en jeu, $\mathrm{n}^{\circ} 17$ (1975) :37-62; Jean-Jacques Nattiez, Musicologie générale et sémiologie, Collection « Musique/Passé/Présent " (Paris : Christian Bourgois, 1987).

${ }^{11}$ Imberty s'inspire ici de la thèse de Didier Anzieu (responsable de la collection « Psychismes » et auteur de plusieurs ouvrages, parmi lesquels on retrouve L'auto-analyse de Freud [Paris : Presses universitaires de France, 1975]), selon laquelle les processus inconscients sont limités; ils sont les mêmes partout et toujours, donc universels.
} 
le niveau poïétique avec la certitude que les processus psychiques découverts au niveau esthésique et incarnés au niveau neutre se manifestent aussi lors de la création.

\section{DU NIVEAU ESTHÉSIQUE À LA DÉDUCTION DU NIVEAU NEUTRE}

Pour Michel Imberty, la musique est un système symbolique qui repose sur une structure intentionnelle de sens, ce sens étant une réalité psychologique complexe qui ne se manifeste pleinement que lorsqu'elle est reçue par l'auditeur. On trouve au long du texte cinq définitions du symbolique : les deux premières touchent le symbole en général et les trois autres se réfèrent à la particularité du symbole musical :

1. Rapport, naturel ou conventionnel, d'analogie, de ressemblance ou d'imitation du symbolisant au symbolisé, qui repose sur une communauté de réactions affectives suscitées par le symbolisant et le symbolisé chez l'utilisateur du symbole (p. 4).

2. Substitut psychique de l'objet (p. 11).

3. Représentation et imitation d'un dynamisme temporel (p. 12).

4. Figuration dynamique qui draine les charges affectives de relations psychiques les plus centrales (p. 47).

5. Incarnation sonore d'un processus symbolique qui exprime le fonctionnement des schèmes psychiques de l'intégration et de la désintégration du Moi (p. 48).

Il existe aussi des définitions complémentaires : celle de la fonction symbolique en tant que déformation subjective du réel et négation de la réalité immédiate; et celle de l'activité symbolique en tant qu'investissement psychique ${ }^{12}$ et omniprésence du sujet. Le symbole, incarnation des processus psychiques inconscients, appelle l'interprétation, qui suit l'idéal de l'authenticité. Imberty fait le parallèle entre l'interprétation musicale et l'interprétation psychanalytique, car il s'agit de la même démarche, de la même recherche.

Interprétation musicale authentique : Activité de la conscience intentionnelle. Relation de « résonance » psychique entre l'œuvre et l'interprète. Coïncidence absolue entre l'introjection du non-dit de l'œuvre et de son style, et le mouvement de projection de l'interprète vers elle (p. 34).

\footnotetext{
${ }^{12}$ L'expression « investissement psychique » est d'une importance capitale, car elle exprime clairement le point de vue psychanalytique qui caractérisera les interprétations du symbole musical chez Imberty. Il s'agit d'un processus qui enracine la perception et la compréhension de l'œuvre dans l'inconscient. La relation œuvre-sujet serait alors une relation du sujet avec lui-même à travers l'œuvre. L'œuvre n'existe que par les processus d'investissement : projection, introjection, identification projective, refoulement, sublimation.
} 
Interprétation psychanalytique authentique : Résonance affective et conceptuelle particulière. Correspondance entre l'introjection de l'œuvre et la projection de l'interprète analyste. Restitution du processus intentionnel qui lie l'œuvre à son public (p. 38-39).

D'une façon semblable, et grâce à l'authenticité d'une écoute disponible, l'auditeur réalise alors une représentation sémantique du style : véritable décryptage intellectuel, perceptif et affectif de l'œuvre lors de l'audition; ensemble de significations potentielles de l'œuvre; structure sémantique latente des réponses verbales associées par les auditeurs à l'œuvre, et qui représente ce qu'ils ont retenu de l'intention créatrice qu'elle manifeste (p. 44-45).

L'expérience de représentation sémantique du style musical est exposée de façon détaillée dans Entendre la musique ${ }^{13}$ : les auditeurs ont associé un nombre illimité d'adjectifs à des extraits des Préludes pour piano de Claude Debussy et à des extraits des Intermezzi et des Fantasiestücke pour piano de Johannes Brahms. La rigueur méthodologique caractérise cette expérience : analyse mathématique des correspondances, c'est-à-dire analyse dimensionnelle et analyse topologique; puis interprétation des topologies touchant le contenu des réponses verbales. Les résultats de l'étude de la représentation sémantique chez Brahms et Debussy démontrent l'existence de deux univers sémantiques différents. Dans le cas de Debussy, il s'agit d'un univers d'angoisse et de mort, fortement contrasté, distendu entre les pôles antinomiques de l'intégration et de la désintégration. Le thème essentiel de la représentation sémantique des extraits de Debussy est l'eau, image archétypique et mythasmatique ${ }^{14}$ fondamentale. Trois images de l'eau (stagnante, claire et démontée) sont associées à trois images de la mort (mort d'avant la vie ou originelle, vie, mort d'après la vie ou cosmogonique) (p. 54-65). Dans le cas de Brahms, l'univers sémantique est ambivalent. La nostalgie (immobilité, tendresse, mystère, délicatesse) en serait le thème fondamental. Il se produit chez lui un nouvel élargissement, sur le plan musical, des notions d'intégration et de désintégration. Au contraire de Debussy, qui travaille avec des matériaux contrastants et bien définis sur le plan des antinomies intégration-désintégration, Brahms tend à unifier les contraires, à les concilier, en créant ainsi un univers sémantiquement ambigu (p. 67-76).

L'étude de la représentation sémantique du style constitue la première étape vers la recherche de structures stylistiques ayant conditionné les réponses des auditeurs, pas capital vers la réflexion à propos du niveau neutre. Le temps est la notion clé qui permet d'établir un lien entre la représentation sémantique du style et les structures stylistiques. La définition de résonance émotionnelle explique ce phénomène, car, dans la musique, la résonance émotionnelle n'est . possible que dans le dynamisme, et ce lien essentiel avec le temps permet à la musique de représenter en tant que système symbolique des expériences qui synthétisent la vie psychique du sujet (p. 12).

\footnotetext{
${ }^{13}$ Voir les rappels des expériences dans Imberty, Les écritures du temps, 52, 66.

${ }^{14}$ L'image archétypique concẹrne les paradigmes culturels représentant une même expérience vécue, tandis que l'image mythasmatique fait allusion aux résidus d'une fantasmatique collective.
} 
Pour découvrir de quelle façon l'œuvre musicale représente le temps, Imberty utilise une opération logique de l'analyse sémiologique, la segmentation, qui devient dans sa démarche un processus perceptif réel permettant de décoder la forme. Il s'agit de la perception des changements qualitatifs dans le flux temporel, perception qui dépend des modèles de référence et qui témoigne en même temps de la structure profonde de l'œuvre et de la façon dont le style est décodé par le sujet (p. 86). De cette opération se dégagent quelques notions d'une grande importance pour l'étude de la structure temporelle de la musique : dynamisme temporel, dynamisme spécifique de la forme, durée musicale, conduites perceptives de décodage, changement, macrostructure, vecteurs dynamiques, schèmes d'ordre, schèmes de relation d'ordre, points de condensation. Le contenu de ces notions peut être résumé ainsi.

Dynamisme temporel

Dynamisme spécifique de la forme

Durée musicale

Changement

Macrostructure

Vecteurs dynamiques

Schèmes d'ordre

Schèmes de relation d'ordre
Répartition dans le temps des moments de tension et de détentes liés à la charge affective (p. 12).

Évolution de la forme dans le temps (p.11).

Imitation, par une représentation dynamisée, des sentiments d'unité ou de morcellement du Moi (p. 13).

Notion temporelle et structurale. Perception d'une relation entre l'avant et l'après, succession contrastive introduisant dans le flux continu du temps une rupture (p. 87).

Schéma de structuration du temps. Perception et rétention mnémonique de quelques changements particulièrement prégnants, déterminant pour l'auditeur la progression globale de la pièce (p. 90).

Éléments musicaux véhiculant des significations temporelles d'orientation, de progression, de diminution ou de croissance, de répétitions ou de retours (p. 91).

Simples successions et juxtapositions. Relations proches et lointaines : croissance, diminution, répétition, imitation (p. 91).

Relations organiques permettant d'établir des rapports entre les parties temporelles 
proches : thème, variation de thèmes, relations syntaxiques ou rhétoriques (p.91).

Points de condensation

Moment précis où l'auditeur, de manière inconsciente ou implicite, pressent le schéma général temporel, la direction essentielle (p. 104-5).

La macrostructure englobe le fonctionnement des autres paramètres : schèmes d'ordre et de relation d'ordre, vecteurs dynamiques et points de condensation. Elle rappelle le concept de structure d'ordre supérieur chez Ingarden et permet d'aller chercher des visions générales du temps projetées par les sujets compositeur, auditeur - dans les œuvres. La macrostructure possède un double statut : psychologique et culturel. Le statut psychologique répond au fait que la macrostructure est le résultat d'une coordination de schèmes perceptifs temporels qui assimilent la réalité perçue et réorganisent le devenir à partir des points privilégiés de condensation (p. 106). Le statut culturel provient du fait que la macrostructure reflète des stéréotypes culturels et que la connaissance de ces stéréotypes aide notablement à sa constitution lors de la perception. Un exemple souvent cité est celui de la structure tripartite qui, à partir du développement du système tonal, devient une espèce d'archétype formel de la musique occidentale. Le schème " exposition-crise-résolution » est une forme de représentation symbolique du temps propre à la culture occidentale.

Imberty réalise deux expériences, toujours avec des extraits de Brahms et de Debussy : la segmentation auditive et la mémorisation de la macrostructure (p. 96-99). Ces expériences cherchent à montrer quelles relations existent entre le contenu des représentations sémantiques du style (expérience précédente) et l'organisation intrinsèque du temps musical. Dans le cas de Debussy, la dissymétrie de la forme, la faible hiérarchie perceptive des changements et, en général, de la macrostructure, la pluralité des points de condensation, les juxtapositions, les ruptures, les contrastes, l'immobilisation progressive du devenir et le morcellement du temps déséquilibrent le climat émotionnel, renvoient aux expériences destructrices du Moi et provoquent le déni du temps. Cela explique l'univers sémantique dominé par les idées de l'angoisse et de la mort. Dans le cas de Brahms, la forte hiérarchie des changements, la symétrisation du devenir et la présence d'un point de condensation qui organise la totalité, créent un univers continu, progressif, ordonné, symétrique, hiérarchisé, où des sentiments opposés peuvent alterner sans conflit, un univers musical qui exprime une expérience équilibrée du temps. Une troisième expérience, réalisée avec des musiciens professionnels, vient s'ajouter (p.119-22) : elle consiste à décrire les séquences spécialement intéressantes du point de vue des vecteurs dynamiques. Les réponses démontrent que les vecteurs dynamiques n'ont pas d'objectivité immédiate, mais qu'ils sont les témoins d'une intention de sens perçue en tant que mouvement de la forme en devenir. 


\section{DU NIVEAU ESTHÉSIQUE À LA DÉDUCTION DU NIVEAU PÖ̈ÉTIQUE}

Une des hypothèses de l'ouvrage d'Imberty est la suivante : par un mimétisme réciproque du temps du Moi et du temps musical, se dégage une composante sémantique du style qui renvoie aux processus inconscients de l'activité représentative symbolique (p.95). La démarche interprétative du psychanalyste serait donc de déchiffrer les symboles du temps dans le style des œuvres :

[...] la sémantique musicale est donc une sémantique du temps, des schèmes temporels et des expériences de la vie intérieure et du Moi impliquées par le temps. Plus profondément, si le temps est aussi essentiel à l'expressivité musicale, c'est qu'il met en jeu, au niveau de l'inconscient, les pulsions de vie et de mort (p. 149).

Une fois arrivée à la thèse que le style musical, organisation temporelle spécifique de l'œuvre, est une représentation symbolique des fantasmes du temps, la psychanalyse peut proposer une interprétation. Brahms, représentant du temps romantique et de l'alternance cyclique, élabore une position dépressive basée sur l'ambivalence des sentiments propres et de leur intégration au Moi (p.156). Debussy, par le morcellement du temps et la désintégration, élabore une position paranoïde-schizoïde (p.155). Le temps fantasmatique chez Brahms exprime une intégration organique, un flux continu, une forme musicale fermée sur ellemême. C'est une fantasmatique qui accepte l'écoulement du temps et de la destinée, qui a conscience de la mort et l'accepte. Imberty explique l'utilisation des formes classiques chez Brahms par la nécessité de superposer au temps existentiel irréversible une architecture symétrique qui donne une représentation acceptable de la mort (p.157). Debussy, au contraire, est dominé par la pulsion de mort. C'est un temps dénié, un temps de mort où le hasard et l'accident bloquent le devenir, et qui reflète des mythasmes de la culture symboliste tout en révélant les fantasmes debussystes (p.154-55). Imberty utilise le concept de crise du milieu de la vie, prise de conscience de la mort, réélaboration de la position dépressive qui se produit entre les âges de 30 et de 40 ans. Chez les créateurs, cette crise s'exprime par un ralentissement du rythme productif, par un travail méticuleux et un changement de style qui révèle si la crise a été ou non surmontée. Chez Brahms, il y a acceptation de la mort, donc, crise surmontée, tandis que c'est le contraire chez Debussy (p. 160-62). La mort serait alors l'inquiétude de toute vie. Imberty écrit d'ailleurs : « La musique n'aurait donc été inventée par les hommes que pour contourner la mort, écrire le temps des angoisses et des peurs qui minent la fragilité de l'être. Et pourtant, combien de plaisirs ineffables, combien de joies! » (p. 237).

\section{LE TEMPS RETROUVÉ}

Le passage du niveau esthésique aux niveaux neutre et poïétique se poursuit et s'achève par une interprétation des moments cruciaux de l'histoire de la musique. On peut résumer ainsi les idées qu'Imberty met en valeur. 
Monodie florentine

L'ethos harmonique

Beethoven

Wagner

$\mathrm{XX}^{\mathrm{e}}$ siècle
Dynamisme pur de la mélodie. Mouvements de l'âme. Temporalité de l'expression vocale qui retrouve le temps humain de la vie et des sentiments (p.179).

Autonomie signifiante par la syntaxe musicale. Temps clos, conceptualisé, architecture réversible s'inscrivant dans un schème tripartite : tonalité principale, modulations, retour à la tonalité principale. Image fantasmatique de l'homme mûr, du Moi fort et intégré, sûr de soi face à la mort (p. 183-90).

Le temps de l'agir, développement de l'action du Moi sur le monde. Historicité. Temps orienté vers un avenir valorisé par l'accomplissement supérieur du Moi, dans la lutte contre tous les obstacles à surmonter. Temps de l'ascendance et de la réparation intégrative (p.19397).

Temps virtuel. Devenir irréversible, continu, rapports toujours nouveaux, irrationalité de la conscience romantique. Structures de renvois à l'inactuel, à l'inentendu, à l'inaccessible et l'indéterminé. Fantasme de l'errance et de la quête de l'absence (p. 198-207).

Temps désintégré. Instant du bruit ou du son pur. Désintégration du Moi (p. 212-13).

Jusqu'à la fin du romantisme, la musique occidentale se caractérise par l'intégration et l'unité du devenir. Debussy serait un des premiers à exprimer l'impossibilité de dominer le temps. Ce passage du temps unifié au temps discontinu peut être expliqué du point de vue de la psychanalyse par un retour à la conception la plus archaïque du temps. En fait, le Moi archaïque est un moi atemporel, car, à la naissance, "l'enveloppe sonore du Soi » est ponctuelle, pleine de silences et de ruptures, et non pas continue ou unifiée. Ce n'est que plus tard, grâce à l'échange avec l'environnement, que l'identité et l'identification apparaissent, et qu'avec elles surgit le temps continu et unifié (p. 215). Dans cette perspective, un moment crucial dans l'histoire de la musique est représenté par les œuvres de Claudio Monteverdi, dont la construction met en relief des procédés comme l'écho, qui reflètent un temps transitionnel. Chez Debussy, les réminiscences et les duplications seraient (aussi) une forme d'écho, la symbolique musicale devient plus archaïque et elle est dominée par la pulsion de mort :

L'œuvre de Debussy inaugure les représentations stylistiques du temps contemporain qui est le temps de Thanatos : le devenir y est bloqué, éclaté 
en mille instants qui ne durent pas, alternances de surgissements chaotiques et d'immobilités glacées. [...] La musique contemporaine, à partir de Varèse, fait de cette désorganisation organisée et du morcellement du temps et de la substance sonore l'essentiel de son esthétique (p. 227-28).

Une des notions qui expliquent l'attitude de l'homme et de l'artiste contemporain devant la mort est celle de l'invention paradoxale :

J'entends par invention paradoxale, une invention où le compositeur crée l'œuvre non pour qu'elle soit, mais pour qu'elle reste indéfiniment à faire, y laissant une part importante soit à l'interprète [...], soit au hasard ou à l'automatisme algébrique de l'écriture. [...] L'invention paradoxale me semble une figure de la pulsion de mort [...] (p. 229).

Donc, l'œuvre n'accomplit plus, dans la musique contemporaine, sa fonction de détournement de l'angoisse. Les caractéristiques de notre époque - destruction des distinctions psychiques fondamentales, confusion de rôles sociaux et des valeurs culturelles - mettent en relief le fait que la séparation entre la réalité interne et la réalité externe est floue. C'est une période archaïque où la pensée se tourne vers l'imaginaire; dans cette perspective, l'ambiguïté de la société contemporaine serait donc une contestation de la rationalité. Mais cette situation archaïque donnera naissance à des réalisations artistiques qui tenteront encore une fois l'unité du Moi, l'intégration. C'est avec ce rêve du temps retrouvé que Michel Imberty couronne son ouvrage :

[...] des sons épars, filtrés, disséqués, décomposés, des bruits trafiqués, des
durées insaisissables, des timbres et des densités, émerge lentement une
mélodie plus pure et plus solitaire, qui fond soudain tout ce bric-à-brac
dérisoire dans une éphémère unité. Bruits du monde, bruits du corps qui
soudain retrouvent leur signifiance, comme dans cette Sequenza III
prémonitoire de Luciano Berio, où l'immense vocalise mystique [...] s'élance
dans l'espace ouvert,s'étire infiniment dans le temps musical retrouvé (p.234).

\section{EN GUISE DE CONCLUSION : RÉFLEXIONS CRITIQUES}

Fermons le cercle et retournons à l'hypothèse de départ de l'ouvrage, exposée déjà dans l'introduction de cet essai. Elle est sans doute son point central et son fil conducteur - presque un ostinato. À présent, il est possible de présenter les étapes qui forment l'énonciation de ce postulat : le style musical est une écriture du temps qui donne à l'homme l'illusion de pouvoir contourner la mort (p. ix).

Style musical Structure temporelle créée et perçue (p. xiii).

Temps

Mimétisme entre le temps musical et le temps du Moi, par opposition au temps réel qui représente le vieillissement et la mort (p.164). 
Mort

Les vécus internes du temps sont en rapport avec le phénomène de la mort et du vieillissement. L'œuvre musicale, en tant que dessin temporel, permet de contourner la mort (p. 149, 174).

La construction de ce postulat part de la prémisse d'ordre psychanalytique que tout le vivant consiste à contourner la mort; ce qui semble correspondre à la culture occidentale, surtout à partir de la Renaissance, mais qui serait discutable pour d'autres visions du monde.

Affirmer que la fonction principale de la musique serait de consoler l'être humain de la peur de la mort et du vieillissement voudrait dire que, dans le cas d'une compréhension de ces phénomènes la musique, "métaphysique compensatoire », telle qu'Adorno l'a définie, disparaitrait, ce qui est sans doute une hypothèse problématique qui risque d'être mise en question par les cultures qui ont intégré le vieillissement et la mort dans leur vécu social, qui ne les ont jamais évacué ou fui, et qui possèdent une tradition musicale indiscutable.

La démarche interprétative particulière d'Imberty et l'approche psychanalytique en général réalisent un saut spéculatif qui dépasse une certaine barrière ou limite importante, et cela non d'une façon graduelle, mais brusquement. Comme exemple concret, on pourrait citer celui de la représentation sémantique du style de Debussy. Il s'agit de l'analyse de l'image de l'eau comme paradigme de réponses données par les auditeurs. Il est pertinent de remarquer que l'eau est le symbole fondamental de la musique de Debussy et de tout le mouvement symboliste, et que ce symbole a été découvert par les auditeurs, ce qui prouve, du moins à une certaine échelle, que le symbole est l'incarnation de processus psychiques universels. Or, affirmer que les trois images de l'eau correspondent aux trois images de la mort est un saut trop grand qui entre en contradiction avec la rigueur méthodologique d'Imberty.

Prenons les questions critiques de Jean Molino à propos des lectures psychanalytiques :

Le problème herméneutique posé par ces démarches - nous laissons de côté ici le problème de leur validité « scientifique »- est double : en premier lieu, qu'est-ce donc qu'une signification inconsciente? On peut parler en effet de conséquences non intentionnelles d'un acte, mais de quel droit parler d'une signification non consciente, d'un sens non voulu? À supposer même qu'il soit légitime d'accepter une telle notion, il faudrait la préciser suffisamment pour que l'on dégage les caractères qui la distinguent de la signification intentionnelle, au sens courant du mot ${ }^{15}$.

Chez Imberty, la fusion intentionnel-inconscient est totale. La recherche de - l'intentionnalité est en même temps la recherche des processus inconscients universels. La terminologie définissant la frontière entre ce qui est conscient et

${ }^{15}$ Molino, « Pour une histoire de l'interprétation : les étapes de l'herméneutique (suite) », Philosophiques 12, $\mathrm{n}^{\circ} 2$ (automne 1985) : 290. 
ce qui est inconscient est floue, et on ne saurait penser autrement que Molino : "Rien n'est plus dangereux que d'assimiler, plus ou moins clairement, une signification inconsciente à une signification consciente $[. . .]^{16}$. » Cependant, la critique la plus importante que l'on peut adresser à l'herméneutique d'Imberty est sans doute la fusion des trois niveaux de la tripartition : poïétique, neutre et esthésique, toujours à la recherche d'un sens unique et universel. La confusion des niveaux et la croyance en l'unicité du sens sont les causes fondamentales des conflits multipliés de l'herméneutique moderne. Molino a très lucidement mis l'accent sur ce fait : «Les apories de l'herméneutique naissent de la confusion de ces trois dimensions; ou, plus exactement, l'analyste entend, à partir de l'une des dimensions, rendre compte des autres $[\ldots]^{17}$ », ce qui est exactement le cas d'Imberty. Molino, qui part de la définition de l'interprétant chez le philosophe Charles Sanders Peirce et voit le signe comme un renvoi infini d'un signe à un autre, affirme : «Une fois que l'on a reconnu cette multiplicité et cette hétérogénéité des significations, les apories de l'herméneutique disparaissent $[\ldots]^{18}$. Il n'y a pas une signification, mais des significations, et cela est la seule façon de rendre compte de l'art en tant que fait social ou fait anthropologique total. Malgré la rigueur de la démarche d'Imberty, sa position théorique demeure dans la situation ambiguë et confuse qui caractérise l'herméneutique contemporaine.

Malgré l'effort évident de créer un corpus catégoriel précis, il persiste dans Les écritures du temps des problèmes importants. Le premier est celui de l'ambiguïté des notions de temps et de durée - possible héritage des approches phénoménologiques. Souvent, les deux notions vont apparaître fusionnées, indifférenciées, associées; l'une peut même remplacer l'autre; et pourtant, dans le contexte précis de la musique, il s'agit de deux concepts complètement différents. Le temps est une catégorie philosophique, abstraite, tandis que la durée est un paramètre limité, spécifique. La création d'une forme dans le temps, le dessin temporel que fait la musique sur l'écoulement du temps réel, ne peut concerner que le temps en tant que catégorie philosophique, en tant que dimension abstraite, en tant qu'élément d'une vision du monde. La durée, mesure trop concrète du temps, ne saurait jamais expliquer la complexité du phénomène qu'Imberty veut mettre en relief. Le deuxième concerne la notion de style, vaste et complexe, mais aussi imprécise, ce qui conduit à très peu de distinction entre les définitions du style musical et celles de la musique en tant qu'art.

Il existe dans Les écritures du temps une fusion énigmatique de ces deux concepts. Est-ce que musique et style musical constituent le même phénomène? Est-ce que style individuel, style d'une époque ou style d'une culture correspondent toujours à la même définition de style? L'opposition pensée logiquepensée symbolique, abordée aussi plus tard dans la relation rationalitéimaginaire est aussi un point assez discutable, car Imberty soutient plutôt leur opposition que leur complémentarité. Pour lui, l'art appartient complètement

\footnotetext{
${ }^{16}$ Ibid., 301.

${ }^{17}$ Ibid., 300.

${ }^{18}$ Ibid., 302.
} 
au domaine du symbolique et de l'imaginaire, et les éléments rationnels ne sont là que pour accomplir un rôle secondaire. Mais la division est-elle si nette entre ces deux modes de pensée? Y a-t-il deux modes de pensée différents dont un correspondrait aux arts et l'autre aux sciences? Nous préférons de notre côté aborder ce problème du point de vue de la complémentarité, de l'unité dialectique des processus de pensée autant pour les arts que pour les activités logiques.

Retournons à l'apport capital de la démarche de Michel Imberty telle qu'elle se présente dans Les écritures du temps. L'étude du style musical en tant que structure temporelle et de la relation de la musique, dessin sonore dans le temps, avec les différentes visions du temps correspondant à chaque culture, à chaque époque, et même à chaque individu, et avec les diverses attitudes devant la mort, constitue un pas fondamental pour la compréhension de la spécificité de la musique. Comme dans les processus psychiques hors-temps observés par Marcel Proust, ce qui est essentiel dans la musique est qu'elle permet à l'être "d'obtenir, d'isoler, d'immobiliser - la durée d'un éclair - ce qu'il n'appréhende jamais : un peu de temps à l'état $\operatorname{pur}^{19}{ }^{\text {». }}$

\section{Résumé}

L'ouvrage Les écritures du temps (1981) de Michel Imberty est d'une importance capitale pour la musicologie contemporaine. De la psychologie expérimentale à la psychanalyse, Imberty développe une approche perceptive du style musical dont le but est l'interprétation de la musique en tant que symbolique du temps. Sa démarche est explicable à partir de la sémiologie musicale, qui en clarifie les principes. Imberty construit, avec précision et intelligence, une méthodologie pour l'observation du temps musical, et cela constitue son grand apport à la musicologie contemporaine. Cet essai cherche à mettre en relief les notions et postulats fondamentaux de sa pensée.

${ }^{19}$ Proust, À la recherche du temps perdu : Le temps retrouvé II, 15. 